\title{
Cerebellar pathology and disability worsening in relapsing- remitting multiple sclerosis: A retrospective analysis from the CombiRx trial
}

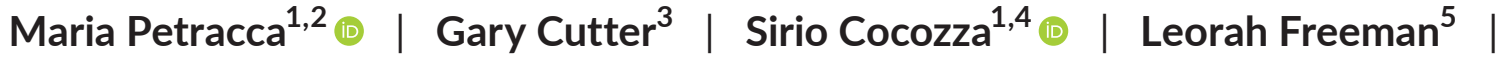 \\ John Kangarlu $^{1}$ | Monica Margoni, ${ }^{1,6}$ | Matteo Moro ${ }^{1,7}$ | Stephen Krieger ${ }^{1}$ | \\ Mohamed Mounir El Mendili ${ }^{1,8}$ | Amgad Droby ${ }^{1,9,10,11}$ | Jerry S. Wolinsky ${ }^{12}$ | \\ Fred Lublin $^{1}$ | Matilde Inglese M,13,14 $^{1,1}$ \\ ${ }^{1}$ Department of Neurology, Icahn School of Medicine at Mount Sinai, New York, New York, USA \\ ${ }^{2}$ Department of Human Neurosciences, Sapienza University, Rome, Italy \\ ${ }^{3}$ Department of Biostatistics, University of Alabama at Birmingham, Birmingham, Alabama, USA \\ ${ }^{4}$ Department of Advanced Biomedical Sciences, University "Federico II", Naples, Italy \\ ${ }^{5}$ Department of Neurology, Dell Medical School, The University of Texas at Austin, Houston, Texas, USA \\ ${ }^{6}$ Padova Neuroscience Centre, University of Padua, Padua, Italy \\ ${ }^{7}$ Department of Informatics, Bioengineering, Robotics and Systems Engineering (DIBRIS), University of Genova, Genova, Italy \\ ${ }^{8}$ Aix Marseille Univ, CNRS, CRMBM, Marseille, France \\ ${ }^{9}$ Laboratory for Early Markers of Neurodegeneration (LEMON), Neurological Institute, Tel Aviv Sourasky Medical Center, Tel Aviv, Israel \\ ${ }^{10}$ Sackler Faculty of Medicine, Tel Aviv University, Tel Aviv, Israel \\ ${ }^{11}$ Sagol School for Neuroscience, Tel Aviv University, Tel Aviv, Israel \\ ${ }^{12}$ University of Texas Health Science Center at Houston (UTHealth), Houston, Texas, USA \\ ${ }^{13}$ Department of Neuroscience, Rehabilitation, Ophthalmology, Genetics and Maternal Child Health, University of Genoa, Genoa, Italy \\ ${ }^{14}$ Ospedale Policlinico San Martino, Istituti di Ricovero e Cura a Carattere Scientifico (IRCCS), Genoa, Italy
}

Correspondence

Matilde Inglese, Department of

Neuroscience, Rehabilitation,

Ophthalmology, Genetics and Maternal

Child Health, University of Genoa, Genoa,

Italy.

Email: m.inglese@unige.it

Funding information

CombiRx (NCT00211887) was funded by the NIH, National Institute of Neurological Disorders and Stroke (phase III study: UO1NS045719)

\begin{abstract}
Background and purpose: Cerebellar damage is a valuable predictor of disability, particularly in progressive multiple sclerosis. It is not clear if it could be an equally useful predictor of motor disability worsening in the relapsing-remitting phenotype.

Aim: We aimed to determine whether cerebellar damage is an equally useful predictor of motor disability worsening in the relapsing-remitting phenotype.

Methods: Cerebellar lesion loads and volumes were estimated using baseline magnetic resonance imaging from the CombiRx trial $(n=838)$. The relationship between cerebellar damage and time to disability worsening (confirmed disability progression [CDP], timed 25-foot walk test [T25FWT] score worsening, nine-hole peg test [9HPT] score worsening) was tested in stagewise and stepwise Cox proportional hazards models, accounting for demographics and supratentorial damage.

Results: Shorter time to 9HPT score worsening was associated with higher baseline Expanded Disability Status Scale (EDSS) score (hazard ratio [HR] 1.408, $p=0.0042$ ) and higher volume of supratentorial and cerebellar T2 lesions (HR $1.005 p=0.0196$ and HR 2.211, $p=0.0002$, respectively). Shorter time to T25FWT score worsening was associated with higher baseline EDSS (HR 1.232, $p=0.0006)$. Shorter time to CDP was
\end{abstract}


associated with older age (HR 1.026, $p=0.0010$ ), lower baseline EDSS score (HR 0.428, $p<0.0001$ ) and higher volume of supratentorial T2 lesions (HR 1.024, $p<0.0001$ ).

Conclusion: Among the explored outcomes, single time-point evaluation of cerebellar damage only allows the prediction of manual dexterity worsening. In clinical studies the selection of imaging biomarkers should be informed by the outcome of interest.

KEYWORDS

cerebellar damage, clinical trials, magnetic resonance imaging, manual dexterity, multiple sclerosis

\section{INTRODUCTION}

Cerebellar atrophy is relevant to clinical disability in multiple sclerosis (MS) [1]. In progressive MS, atrophy of specific cerebellar lobules explains different aspects of motor and cognitive disability [2,3] and cerebellar atrophy is a significant predictor of sustained disability [4]. In the relapsing-remitting (RR) phenotype, a cross-sectional analysis has identified a relationship between cerebellar volume, fatigue and depression [5]. Moreover, several studies, conducted in mixed samples including RRMS patients, suggest a relationship between cerebellar atrophy and motor and cognitive disability [6-12], with a recent study showing a significant role for baseline cerebellar gray matter (GM) volume as predictor of 1-year Expanded Disability Status Scale (EDSS) score worsening [13]. Although cerebellar atrophy has been suggested as a potential short-term imaging metric to monitor treatment effect in primary-progressive MS [4], it is not clear if cerebellar volume could be an equally useful predictor of motor disability worsening, as commonly assessed in clinical trials, in RRMS.

As per the role of cerebellar lesions, both cortical and white matter (WM) lesions correlate with clinical disability in RRMS [14-16] however, brainstem and spinal cord lesions, rather than cerebellar lesions, seem to significantly predict EDSS score worsening over time in relapsing-onset MS[17] and clinically isolated syndrome [18].

Given this background, we performed a retrospective analysis in a large cohort of RRMS patients prospectively enrolled in the CombiRx clinical trial[19] to better define the role of cerebellar damage (lesions and volume) as a meaningful biomarker of motor disability worsening in RRMS, while accounting for the effect of demographic variables and supratentorial damage.

\section{METHODS}

\section{Study design}

We conducted a retrospective analysis of magnetic resonance imaging (MRI) and clinical data from the CombiRx trial (NCT00211887). CombiRx was a 3-year, double-blind, multicenter randomized clinical trial that tested the efficacy of a combined therapy of interferon$\beta 1$ a plus glatiramer acetate versus the efficacy of either agent alone, and provided for double-blind therapy until the last participant completed the 3-year trial, allowing up to 7 years of observation of some participants.

Inclusion criteria for the trial were: confirmed diagnosis of RRMS (by Poser or McDonald criteria) with two or more exacerbations in the prior 3 years; age between 18 and 60 years; EDSS score of 0 to 6; and relapse-free status for at least 30 days preceding screening and randomization. Patients who completed the 3-year placebocontrolled core study were eligible for inclusion in the extension phase, with up to 7 years of total follow-up. As per trial protocol, participants were followed every 12 weeks with neurological assessments. EDSS examinations occurred every 12 weeks in the core study through month 42 and every 6 months thereafter in the extension. The Multiple Sclerosis Functional Composite assessment was performed quarterly in the core study and annually in the extension study. MRI was performed at study entry, months 6, 12, 24, 36, 48 and 60 , and at the end of study visit in the extension.

In the present study, we investigated the role of baseline supratentorial and cerebellar pathology as predictors of time to disability worsening over the CombiRx study period (core study + extension phase).

The trial protocol and its amendments received approval from the applicable central or institutional review boards and the National Institutes of Health (NIH)-appointed study data and safety monitoring committee before site initiation and recruitment of participants. Informed consent was obtained prior to any screening procedures or enrollment.

\section{Clinical outcomes of interest}

Disability worsening was assessed in terms of: (i) time to confirmed disability progression (CDP), defined as a 1.0 increase in the EDSS score from baseline, when baseline score was $\leq 5.0$; or an increase of 0.5 from baseline, when baseline score was $\geq 5.5$, sustained for 6 months (two successive quarterly visits); (ii) time to first $20 \%$ change in timed 25-foot walk test (T25FWT) score; and (iii) time to first $20 \%$ change nine-hole peg test (9HPT) score.

\section{MRI acquisition and analysis}

The MRI protocol included two-dimensional fluid attenuated inversion recovery, dual-echo turbo-spin echo, and isotropic pre- and 
post-contrast T1-weighted images $(0.94 \mathrm{~mm} \times 0.94 \mathrm{~mm} \times 3 \mathrm{~mm}$ voxel dimension). The three-dimensional T1-weighted images were acquired either with spoiled gradient recalled echo (SPGR) or magnetization prepared rapid gradient echo (MPRAGE) sequences (0.94 $\mathrm{mm} \times 0.94 \mathrm{~mm} \times 1.5 \mathrm{~mm}$ voxel dimension).

Baseline MRI datasets were screened to assess their suitability for volumetric analysis. Of the original 1008 datasets available, 109 were excluded because of incomplete cerebellar coverage or presence of cerebellar artifacts. In the remaining 899 datasets, supratentorial and cerebellar T2 hyperintense and gadolinium-enhancing $(G d+)$ lesion number and volumes, together with $G M$ volumes, were computed as follows. Cerebellar T2-weighted hyperintense lesions and T1-weighted $\mathrm{Gd}+$ lesions were segmented by one experienced observer using a semiautomatic segmentation technique ( $\operatorname{Jim} 7$; Xinapse Systems, West Berghol, UK), deriving both cerebellar lesion volume and number. Supratentorial T2-weighted hyperintense lesions and T1-weighted Gd+ lesion volume and number were computed as the difference between whole-brain lesion volume/number estimated during the trial and cerebellar lesion volume/number.

Cerebellar volumes (cerebellar GM, anterior cerebellum volume, posterior cerebellum volume) were calculated on lesion-filled T1weighted images using the Spatially Unbiased Infratentorial Toolbox (SUIT) version 3.2, implemented in Statistical Parametric Mapping (SPM)12, as described in Cocozza et al. [2]. During this step, 61 datasets were excluded because of cerebellar segmentation failure. Briefly, cerebellar segmentation output was visually assessed by an experienced observer (M.P.) in order to ascertain that all cerebellar lobules were included in the final mask and that no extracerebellar structures (transverse and sigmoid sinuses, brainstem) were erroneously classified as cerebellar tissue. If any of the abovementioned tissue classification errors was noted, the participant was excluded from further analyses. The remaining 838 datasets, constituting the final study population, were further processed to obtain supratentorial brain volumes. Briefly, lesion-filled T1-weighted images were segmented into GM, WM and cerebrospinal fluid using the segmentation tool implemented in SPM12 software (http://www.fil.ion.ucl. ac.uk/spm). For each participant, intracranial volume (ICV) was computed as the sum of GM, WM and cerebrospinal fluid. Supratentorial GM volume was then computed as the difference between wholebrain GM volume and cerebellar GM volume.

\section{Statistical analysis}

Descriptive analyses (means, medians, standard deviations, interquartile ranges, frequency distributions) were performed to assess and describe the study population. As supratentorial and cerebellar volumes both partly depend on individual biological features (ICV), and both are affected by MS pathogenic processes, bivariate correlations between ICV, supratentorial volume and cerebellar volume were tested. Stagewise Cox proportional hazards models were built to assess the effect of baseline MRI features on time to CDP, and time to $20 \%$ T25FWT and 9HPT score worsening, assessing the models at
TABLE 1 Demographic and clinical features of the study population $(N=838)$

\begin{tabular}{ll}
\hline Age, years & $37.70 \pm 9.66$ \\
Women, $n(\%)$ & $607(72.4)$ \\
Disease duration from symptom onset, years & \\
Mean \pm SD & $4.22 \pm 5.53$ \\
Median (IQR) & $2(1-5)$ \\
Disease duration from diagnosis, years & \\
Mean \pm SD & $1.07 \pm 3.11$ \\
Median (IQR & $0(0-1)$ \\
Median (range) EDSS score & $2(0-6)$ \\
T25FWT score & $4.99 \pm 1.70$ \\
9HPT score & $20.17 \pm 4.81$
\end{tabular}

Abbreviations: 9HPT, nine-hole peg test; EDSS, Expanded Disability Status Scale; IQR, interquartile range; SD, standard deviation; T25FWT, timed 25 -foot walk test.

each stage. In stage I, demographic and clinical features (age, gender, baseline EDSS score) were entered, in stage II, supratentorial MRI features (T2 hyperintense lesion volume and number, Gd+ lesion volume and number, supratentorial GM volume) were added and, in stage III, cerebellar MRI features (T2 hyperintense lesion volume and number, Gd+ lesion volume and number, cerebellar GM volume [with total volume as predictor in the models predicting CDP and anterior cerebellar volume as predictor in the models predicting time to T25FWT/time to 9HPT score worsening]) were added. After each of the three stages, a stepwise forward selection model was used to select variables until no more explanatory power was available for the model. The statistical analysis was conducted in SAS (version 9.4), with $p$ values $<0.05$ considered as statistically significant and with no adjustments for the multiplicity of testing as we considered this a discovery cohort.

\section{RESULTS}

\section{Study population}

The demographic, clinical and MRI characteristics of the study population are shown in Tables 1 and 2.

\section{Relationship between supratentorial and infratentorial volumes}

As expected, supratentorial and cerebellar volumes were correlated. Specifically, bivariate correlations between ICV, supratentorial volume and cerebellar volume are as follows: ICVsupratentorial volume $(r=0.628, p<0.01)$; ICV-cerebellar volume $(r=0.606, p<0.01)$; supratentorial volume-cerebellar volume $(r=0.575, p<0.01)$. 
TABLE 2 MRI features of the study population $(N=838)$

\begin{tabular}{lc}
\hline Supratentorial Gd+ lesions number & $1.74 \pm 4.30$ \\
Supratentorial Gd+ lesions volume, $\mathrm{ml}$ & $0.11 \pm 0.35$ \\
Supratentorial T2 lesions number & $86.49 \pm 55.49$ \\
Supratentorial T2 lesions volume, $\mathrm{ml}$ & $10.52 \pm 11.59$ \\
Cerebellar Gd+ lesions number & $0.03 \pm 0.24$ \\
Cerebellar Gd+ lesions volume, $\mathrm{ml}$ & $0.004 \pm 0.03$ \\
Cerebellar T2 lesions number & $0.92 \pm 1.82$ \\
Cerebellar T2 lesions volume, $\mathrm{ml}$ & $0.12 \pm 0.30$ \\
Supratentorial GM volume, $\mathrm{ml}$ & $562.33 \pm 73.48$ \\
Cerebellar GM volume, ml & $109.78 \pm 11.64$ \\
Anterior cerebellum volume, $\mathrm{ml}$ & $14.35 \pm 1.62$ \\
Posterior cerebellum volume, $\mathrm{ml}$ & $95.43 \pm 10.20$ \\
\hline
\end{tabular}

Note: All values are reported as mean \pm standard deviation.

Abbreviations: $\mathrm{Gd}+$, gadolinium enhancing; GM, gray matter.

\section{Time to 9HPT worsening}

The first stage of the stagewise model identified significant associations between time to 9HPT worsening, female gender and baseline EDSS score ( $p=0.0435$ and $p<0.0001$, respectively). At the second stage, entering supratentorial MRI variables, baseline EDSS score remained significantly associated with time to 9HPT worsening ( $p=0.0028)$, together with age $(p=0.0059)$, volume of supratentorial $\mathrm{Gd}+$ and T2 lesions ( $p=0.0260$ and $p=0.0061$, respectively), and supratentorial GM volume ( $p=0.0401)$. At the third stage, when entering cerebellar MRI variables, anterior cerebellar volume was significantly associated with time to 9HPT worsening $(p=0.0444)$. The stepwise selection confirmed the association between shorter time to 9HPT worsening, higher baseline EDSS score (hazard ratio [HR] 1.408, $p=0.0042$ ) and higher volume of supratentorial T2 lesions (HR 1.005, $p=0.0196$ ), and highlighted an association between higher volume of cerebellar T2 lesions and shorter time to 9HPT score worsening (HR 2.211, $p=0.0002)$.

\section{Time to T25FWT worsening}

The stagewise model identified significant associations between time to T25FWT worsening and baseline EDSS score $(p=0.0032)$. The stepwise selection confirmed the association between shorter time to T25FWT worsening and higher baseline EDSS score (HR $1.232, p=0.0006$ ).

\section{Time to confirmed disability progression}

At the final stage of the stagewise modeling, significant associations between time to CDP, age, and baseline EDSS score were found
( $p=0.0009$ and $p<0.0001$, respectively). Among the supratentorial variables, volume of supratentorial T2 lesions was associated with CDP ( $p=0.0020)$, but the association was lost once number and volume of cerebellar $\mathrm{Gd}+$ lesions were entered into the model ( $p=0.0389$ and $p=0.0223$, respectively). The stepwise selection confirmed the association between shorter time to CDP, older age (HR 1.026, $p=0.0010$ ), lower baseline EDSS score (HR 0.428, $p<0.0001)$ and higher volume of supratentorial T2 lesions (HR $1.024, p<0.0001)$.

\section{DISCUSSION}

Through our retrospective analysis, we set out to explore the relevance of cerebellar lesions and volumes as predictors of different outcomes of motor disability worsening in RRMS. Our results suggest that cerebellar damage, evaluated at a single time point, is a useful predictor of worsening only in selected functional domains (i.e., manual dexterity). Indeed, in our population, supratentorial and cerebellar lesion load were associated with shorter time to 9HPT score worsening, confirming previous findings in patients with progressive MS, where WM damage (brain and cerebellar lesion volumes/WM microstructural damage), significantly predicted 9HPT score in cross-sectional analyses [2,7]. Although atrophy of salient cortical and cerebellar regions might contribute to manual dexterity impairment $[6,10]$, focal lesions disrupting WM tracts involved in hand motor control most likely represent the main pathophysiological substrate of 9HPT score worsening [11,20]. Among clinicodemographic features, baseline EDSS score significantly predicted 9HPT score worsening, in agreement with a recent longitudinal assessment of hand function in patients with MS [21].

Regarding the risk of T25FWT score worsening, this was greater in patients with higher baseline EDSS scores. This is in line with studies investigating the natural history of clinical outcomes in progressive MS [22,23], and can be explained considering the nonlinear relationship between T25FWT and EDSS scores, with the times to T25FWT score worsening increasing exponentially once a threshold of EDSS 5.5 is reached [23]. As for the role of brain and cerebellar MRI metrics, no significant predictor was identified, which is similar to findings in patients with progressive MS and those with relapsingonset MS $[2,6]$. In fact, ambulation worsening and the need for a walking aid are known to be preferentially associated with damage of other districts, such as deep GM and spinal cord [24-26].

Finally, a shorter time to CDP was identified in older patients, which is not surprising given that aging is associated with a reduced structural central nervous system reserve, a delayed and incomplete recovery from relapses and a decline in disease-modifying therapy efficacy [27]. Although patients with lower disability at baseline seemed more likely to reach CDP faster, this finding must be interpreted with caution, as patients at the lower EDSS levels are exposed to more measurement error which, once corrected at subsequent evaluations, would determine an apparent progression not associated with an actual change in their clinical status. Additionally, 
a plateau effect is usually seen with EDSS scores $>4$, which might favor the detection of CDP in the lower EDSS score group. From an MRI perspective, higher lesion load in the supratentorial compartment was associated with shorter time to CDP. Although atrophy is the most widely applied MRI marker of neurodegeneration and predictor of irreversible disability in MS [13,28-30], supratentorial WM lesion density and accrual significantly account for disability and short-term progression in RRMS [31], with WM lesion load being a relevant contributor in machine-learning models predicting disability over a medium-term follow-up [32]. Our finding, although based on a cross-sectional analysis, seems in line with recent work that, classifying distinct MS subtypes based on pathology-derived MRI features, has identified the lesion-led phenotype, characterized by early accrual of lesions, as the subtype with the highest risk of CDP [33]. As for the role of the cerebellum, although in the stagewise model cerebellar lesions did show a significant association with time to CDP, the stepwise selection did not confirm their relevance. Again, this is in line with observations in patients with progressive MS, where changes in cerebellar volume over time, rather than baseline cerebellar metrics, were relevant for prediction of CDP at 1 year [4]. Indeed, the relatively modest contribution of cerebellar features at baseline to the predictive power of our models does not mean this can be assumed to be the case for longitudinal MRI measures.

Overall, baseline clinical features and lesion loads emerge as the most meaningful predictors in our models. Lesion loads, although generally showing poorer correlations with clinical status than atrophy, are less subject to the heterogeneity of the MRI scanners and/ or acquisition protocols influencing consistency in brain atrophy measurements [34], which might partially account for the poor performance of volumetric measures in our analysis.

The present study has some limitations. First, the technical requirements for cerebellar segmentation prevented the inclusion of the full trial population, reducing the power of our analysis. Second, we choose to derive our imaging biomarkers from the baseline MRI acquisition, rather than analyzing MRI changes over time, which have shown a stronger predictive power than cross-sectional assessments [4]. Again, this choice is related to the fact that screening and exclusions of inadequate images from the follow-up examinations would have further reduced our sample and therefore the power of our investigation.

In conclusion, our findings, beyond confirming the role of cerebellar lesions as predictors of manual dexterity worsening over time, further underline the importance, when designing clinical trials, of accurate selection of the candidate imaging biomarkers according to the outcome of interest.

\section{CONFLICT OF INTERESTS}

Dr Petracca reports honoraria from HEALTH\&LIFE S.r.I. and Novartis for speaking and/or travel support. Dr Cutter reports personal fees from Pythagoras Board membership, Brainstorm Cell Therapeutics, Teva Neuroscience, EMD Serono, Novartis, Pfizer, CSL Behring, Avexis Pharmaceuticals, Genzyme, Medimmune/ Viela Bio, Receptos, Biolinerx, Sanofi-Aventis, Galmed, Opko, National Heart,
Lung, and Blood Institute, National Institute of Child Health and Human Development, Vivus, Genentech, Reata Pharmaceuticals, GW Pharmaceuticals, Roche, Orphazyme, Somahlution, Horizon Pharmaceuticals, Reata Pharma, Merck/Pfizer, Klein-Buendel, Click Thera- peutics, Osmotica Pharmaceuticals, Medday, TG Therapeutics, Perception Neurosciences, Celgene, and Recursion Pharmaceuticals. Dr Cocozza reports speaking honoraria from Shire and Genzyme, advisory board honoraria from Amicus, research grants from FISM. Dr Freeman has received grant support from $\mathrm{NIH}$, PCORI, Race to Erase MS, Genentech, and EMD Serono, program sponsorship from Biogen and EMD Serono, consulting fees from Celgene, Biogen and EMD Serono, and has participated in advisory boards for Genentech, Novartis, Celgene and EMD Serono. She has previously served on the Healthcare Provider Council South Central Region of the National MS Society, and currently serves on the NMSS clinical pilot grant review committee as well as the MS Association of America's Healthcare Advisory Council. Dr Margoni reports grants and personal fees from Sanofi Genzyme, Merck Serono, Novartis and Almirall. She was awarded a MAGNIMS-ECTRIMS fellowship in 2020. Dr Krieger reports consulting or advisory work with Biogen, EMD Serono, Genentech, Genzyme, Mallinckrodt, MedDay, Novartis, Octave, Teva and TG Therapeutics, and non-promotional speaking with Biogen, EMD Serono, Genentech and Novartis, and grant and research support from Biogen and Novartis. Dr El Mendili, Dr Droby, Mr Kangarlu and Dr Moro have nothing to disclose. Dr Wolinsky received compensation for consulting, scientific advisory boards, or other activities with Alkermes, Avotres, Brainstorm Cell Therapeutics, Cleveland Clinic Foundation, EMD Serono, GW Pharma, MedDay Pharmaceuticals, NervGen Pharma Corp, Novartis, Roche/Genentech, Sanofi Genzyme and the University of Alabama; royalties are received through UTHealth for out licensed monoclonal antibodies to Millipore (Chemicon International) Corporation. Dr Lublin reports grants and personal fees from Novartis, Biogen Idec, Teva Neuroscience, Sanofi/Genzyme, Celgene, grants from Transparency Life Sciences, and personal fees from Bayer Healthcare, EMD Serono, Actelion, Acorda, Questcor/Malinckrodt, Roche/Genentech, Medimmune, Osmotica, Xenoport, Receptos, Forward pharma, BBB Technologies, Akros, TG therapeutics, Abbvie, MedDay and Atara Biotherappeutics. Dr Inglese reports grants received grants from the NIH, National Multiple Sclerosis Society and FISM, and received fees for consultation from Roche, Genzyme, Merck, Biogen and Novartis.

\section{AUTHOR CONTRIBUTIONS}

Maria Petracca: Conceptualization (supporting); Data curation (lead); Writing - original draft (lead); Writing - review and editing (equal). Gary Cutter: Conceptualization (equal); Data curation (equal); Formal analysis (lead); Investigation (equal); Methodology (equal); Software (equal); Writing - review and editing (equal). Sirio Cocozza: Formal analysis (equal); Writing - review and editing (equal). Leorah Freeman: Investigation (equal); Writing - review and editing (equal). John Kangarlu: Data curation (equal); Formal analysis (supporting); Writing-review \& editing (equal). 
Monica Margoni: Data curation (equal); Formal analysis (supporting); Writing - review and editing (equal). Matteo Moro: Data curation (equal); Formal analysis (equal); Writing - review and editing (equal). Stephen Krieger: Investigation (equal); Writing - review and editing (equal). Mohamed Mounir El Mendili: Data curation (equal); Formal analysis (lead); Methodology (equal); Writing review and editing (equal). Amgad Droby: Data curation (equal); Formal analysis (equal); Writing - review and editing (equal). Jerry S Wolinsky: Conceptualization (equal); Funding acquisition (equal); Investigation (equal); Methodology (equal); Resources (equal); Supervision (equal); Writing - review and editing. Fred Lublin: Conceptualization (equal); Funding acquisition (equal); Investigation (equal); Resources (equal); Supervision (equal); Writing - review and editing. Matilde Inglese: Conceptualization (equal); Funding acquisition (equal); Investigation (equal); Resources (equal); Software (equal); Supervision (equal); Writing - review and editing.

\section{DATA AVAILABILITY STATEMENT}

Anonymized data not published within this article will be made available by reasonable request from any qualified investigator.

\section{ORCID}

Maria Petracca (D) https://orcid.org/0000-0001-9429-2769

Sirio Cocozza (D) https://orcid.org/0000-0002-0300-5160

Monica Margoni (DD https://orcid.org/0000-0002-9232-8964

\section{REFERENCES}

1. Mormina E, Petracca M, Bommarito G, et al. Cerebellum and neurodegenerative diseases: beyond conventional magnetic resonance imaging. World J. Radiol. 2017;9(10):371-388.

2. Cocozza S, Petracca M, Mormina E, et al. Cerebellar lobule atrophy and disability in progressive MS. J Neurol Neurosurg Psychiatry. 2017;88(12):1065-1072.

3. Cocozza S, Pontillo G, Russo C, et al. Cerebellum and cognition in progressive MS patients: functional changes beyond atrophy? J Neurol. 2018;265(10):2260-2266. https://doi.org/10.1007/s0041 5-018-8985-6

4. Inglese $\mathrm{M}$, Petracca $\mathrm{M}$, Mormina $\mathrm{E}$, et al. Cerebellar volume as imaging outcome in progressive multiple sclerosis. PLoS One. 2017;12(4):1-11.

5. Lazzarotto A, Margoni M, Franciotta S, et al. Selective cerebellar atrophy associates with depression and fatigue in the early phases of relapse-onset multiple sclerosis. Cerebellum. 2020;19(2):192-200.

6. Anderson VM, Fisniku LK, Altmann DR, Thompson AJ, Miller DH. MRI measures show significant cerebellar gray matter volume loss in multiple sclerosis and are associated with cerebellar dysfunction. Mult Scler. 2009;15(7):811-817.

7. Anderson VM, Wheeler-Kingshott CA, Abdel-Aziz K, et al. A comprehensive assessment of cerebellar damage in multiple sclerosis using diffusion tractography and volumetric analysis. Mult Scler. 2011;17(9):1079-1087. http://www.ncbi.nlm.nih.gov/pubme $d / 21511688$

8. Weier K, Penner IK, Magon S, et al. Cerebellar abnormalities contribute to disability including cognitive impairment in multiple sclerosis. PLoS One. 2014;9(1):e86916.

9. D'Ambrosio A, Pagani E, Riccitelli GC, et al. Cerebellar contribution to motor and cognitive performance in multiple sclerosis: an MRI sub-regional volumetric analysis. Mult. Scler. 2017;23(9):1194-1203.
10. Grothe M, Lotze M, Langner S, Dressel A. Impairments in walking ability, dexterity, and cognitive function in multiple sclerosis are associated with different regional cerebellar gray matter loss. Cerebellum. 2017. https://doi.org/10.1007/s12311-017-0871-8

11. Cordani C, Meani A, Esposito F, et al. Imaging correlates of hand motor performance in multiple sclerosis: a multiparametric structural and functional MRI study. Mult Scler J. 2020;26(2):233-244.

12. Ruggieri S, Bharti K, Prosperini L, et al. A comprehensive approach to disentangle the effect of cerebellar damage on physical disability in multiple sclerosis. Front Neurol. 2020;11:1-9.

13. Rocca MA, Valsasina $P$, Meani $A$, et al. Association of gray matter atrophy patterns with clinical phenotype and progression in multiple sclerosis. Neurology. 2021;96(11):e1561-e1573.

14. Damasceno A, Damasceno BP, Cendes F. The clinical impact of cerebellar grey matter pathology in multiple sclerosis. PLoS One. 2014;9(5):1-7.

15. Fartaria MJ, O'Brien K, Şorega A, et al. An ultra-high field study of cerebellar pathology in early relapsing-remitting multiple sclerosis using MP2RAGE. Invest Radiol. 2017;52:265-273. http:// content.wkhealth.com/linkback/openurl?sid=WKPTLP:landingpag e\&an=00004424-900000000-99145

16. Tobyne SM, Ochoa WB, Daniel B, et al. Cognitive impairment and the regional distribution of cerebellar lesions in multiple sclerosis. Mult Scler J. 2017;23:1-9.

17. Dekker I, Sombekke MH, Balk LJ, et al. Infratentorial and spinal cord lesions: cumulative predictors of long-term disability? Mult Scler J. 2020;26(11):1381-1391.

18. Tintore M, Rovira A, Arrambide G, et al. Brainstem lesions in clinically isolated syndromes. Neurology. 2010;75(21):1933-1938.

19. Lublin FD, Cofield SS, Cutter GR, et al. Randomized study combining interferon and glatiramer acetate in multiple sclerosis. Ann Neurol. 2013;73(3):327-340.

20. Petracca M, Schiavi S, Battocchio M, et al. Streamline density and lesion volume reveal a postero-anterior gradient of corpus callosum damage in Multiple Sclerosis. Eur J Neurol. 2020;27(6):1076-1082.

21. Newsome SD, von Geldern G, Shou H, et al. Longitudinal assessment of hand function in individuals with multiple sclerosis. Mult Scler Relat Disord. 2020;32:107-113.

22. Koch MW, Mostert J, Uitdehaag B, Cutter G. Clinical outcome measures in SPMS trials: an analysis of the IMPACT and ASCEND original trial data sets. Mult Scler J. 2019;26(12):1540-1549.

23. Koch MW, Metz L, Cutter G. An enrichment strategy for clinical trials in SPMS. Mult Scler J. 2021;27(12):1884-1893.

24. Kearney $\mathrm{H}$, Rocca MA, Valsasina P, et al. Magnetic resonance imaging correlates of physical disability in relapse onset multiple sclerosis of long disease duration. Mult Scler. 2014;20(1):72-80. http:// www.pubmedcentral.nih.gov/articlerender.fcgi?artid $=41077$ 76\&tool=pmcentrez\&rendertype $=$ abstract

25. Nourbakhsh B, Azevedo C, Maghzi AH, et al. Subcortical grey matter volumes predict subsequent walking function in early multiple sclerosis. J Neurol Sci. 2016;366:229-233. https://doi. org/10.1016/j.jns.2016.04.054

26. Jakimovski D, Weinstock-Guttman B, Hagemeier J, et al. Walking disability measures in multiple sclerosis patients: correlations with MRI-derived global and microstructural damage. J Neurol Sci. 2018;393:128-134. https://doi.org/10.1016/j.jns.2018.08.020

27. Zeydan B, Kantarci OH. Impact of age on multiple sclerosis disease activity and progression. Curr Neurol Neurosci Rep. 2020;20(7):1-7.

28. Ruggieri S, Petracca M, De GL, et al. A matter of atrophy: differential impact of brain and spine damage on disability worsening in multiple sclerosis. J Neurol. 2021; (0123456789). https://doi. org/10.1007/s00415-021-10576-9

29. Pontillo G, Cocozza S, Di Stasi M, et al. 2D linear measures of ventricular enlargement may be relevant markers of brain atrophy and long-term disability progression in multiple sclerosis. 2020. 
30. Inglese M, Petracca M. MRI in multiple sclerosis: clinical and research update. Curr Opin Neurol. 2018;31(5):1-7.

31. Gaetano L, Magnusson B, Kindalova P, et al. White matter lesion location correlates with disability in relapsing multiple sclerosis. Mult Scler J - Exp Transl Clin. 2020;6(1):205521732090684.

32. Tommasin S, Cocozza S, Taloni A, et al. Machine learning classifier to identify clinical and radiological features relevant to disease progression in multiple sclerosis. J Neurol. 2021. http:// ovidsp.ovid.com/ovidweb.cgi?T=JS\&PAGE=reference $\& D=$ emexb $\& N E W S=N \& A N=631450572$

33. Eshaghi A, Young AL, Wijeratne PA, et al. Identifying multiple sclerosis subtypes using unsupervised machine learning and MRI data. Nat Commun. 2021;12(1):1-12. https://doi.org/10.1038/s41467021-22265-2
34. Storelli L, Rocca MA, Pantano P, et al. MRI quality control for the Italian Neuroimaging Network Initiative: moving towards big data in multiple sclerosis. J Neurol. 2019;266(11):2848-2858. https://doi. org/10.1007/s00415-019-09509-4

How to cite this article: Petracca M, Cutter G, Cocozza S, et al. Cerebellar pathology and disability worsening in relapsing-remitting multiple sclerosis: $\mathrm{A}$ retrospective analysis from the CombiRx trial. Eur J Neurol. 2022;29:515-

521. https://doi.org/10.1111/ene.15157 